\title{
Hand Impairment in Systemic Sclerosis: Various Manifestations and Currently Available Treatment
}

Amber Young, $M D^{1,2}$

Rajaie Namas, $M D^{1,2}$

Carole Dodge, OTR/L, CHT

Dinesh Khanna, $M D, M S^{1,2, *}$

\author{
Address \\ ${ }^{*}, 1$ Division of Rheumatology, Department of Internal Medicine, University of \\ Michigan, Suite 7C27, 300 North Ingalls Street, SPC 5422, Ann Arbor, 48109, \\ MI, USA \\ Email: khannad@med.umich.edu \\ ${ }^{2}$ Department of Internal Medicine, University of Michigan Scleroderma Program, \\ University of Michigan, Ann Arbor, MI, USA \\ ${ }^{3}$ Division of Occupational and Physical Therapy, University of Michigan, Ann \\ Arbor, MI, USA
}

Published online: 19 July 2016

(C) Springer International Publishing AG 2016

This article is part of the Topical Collection on Scleroderma

Keywords Systemic sclerosis - Scleroderma • Hand involvement - Hand impairment - Arthralgias · Inflammatory arthritis · Puffy hands $\cdot$ Skin sclerosis $\cdot$ Acro-osteolysis $\cdot$ Calcinosis

\section{Opinion statement}

Systemic sclerosis (SSC) is an autoimmune disease initially recognized by hand involvement due to characteristic Raynaud's phenomenon (RP), puffy hands, skin thickening, and contractures resembling claw deformities. SSc contributes to hand impairment through inflammatory arthritis, joint contractures, tendon friction rubs (TFRs), RP, digital ulcers (DU), puffy hands, skin sclerosis, acro-osteolysis, and calcinosis. These manifestations, which often coexist, can contribute to difficulty with occupational activities and activities of daily living (ADL), which can result in impaired quality of life. However, despite this knowledge, most diagnostic and treatment principles in SSc are focused on visceral manifestations due to known associations with morbidity and mortality. Treatment of inflammatory arthritis is symptom based and involves corticosteroids $\leq 10 \mathrm{mg}$ daily, methotrexate, tumor necrosis factor inhibitors, tocilizumab, and abatacept. Small joint contractures are managed by principles of occupational hand therapy and rarely surgical procedures. TFRs may be treated similar to inflammatory arthritis with corticosteroids. All patients with RP and DU should keep digits covered and warm and avoid vasoconstrictive 
agents. Pharmacologic management of RP begins with use of calcium channel blockers, but additional agents that may be considered are fluoxetine and phosphodiesterase 5 (PDE5) inhibitors. DU management also involves vasodilators including calcium channel blockers and PDE5 inhibitors; bosentan has also been shown to prevent DU. In patients with severe RP and active DU, intravenous epoprostenol or iloprost can be used and surgical procedures, such as botulinum injections and digital sympathectomies, may be considered. For those with early diffuse cutaneous SSc needing immunosuppression for skin sclerosis, methotrexate or mycophenolate mofetil can be used, but the agent of choice depends on coexisting manifestations, such as inflammatory arthritis and/or lung involvement. Various pharmacologic agents for calcinosis have been considered but are generally ineffective; however, surgical options, including excision of areas of calcinosis, can be considered. Overall management of hand impairment for all patients with SSC should include occupational hand therapy techniques such as range of motion exercises, paraffin wax, and devices to assist in ADL. Thus, treatment options for the various manifestations contributing to hand impairment in SSC are limited and often modestly efficacious at best. Robust studies are needed to address the manifestations of SSc that contribute to hand impairment.

\section{Introduction}

Systemic sclerosis (SSc) is an autoimmune disease that can affect the gastrointestinal (GI) tract, heart, lungs, kidneys, skin, and/or vasculature through a complex interplay of fibrosis, inflammation, and vascular damage. Given the significant morbidity and mortality associated with progressive skin fibrosis and visceral organ involvement, most published literature and current research is focused on those areas. Ongoing clinical trials at this time are centered on skin sclerosis, interstitial lung disease (ILD), pulmonary arterial hypertension (PAH), Raynaud's phenomenon (RP), and digital ulcers (DU). However, only minimal evidence is available on therapy and patient outcomes for hand impairment in SSc.

Hand impairment is almost universal in SSc. Disease manifestations of SSc that contribute to hand impairment include inflammatory arthritis, tendon friction rubs
(TFRs), tendonitis/tendinosis, puffy hands, skin sclerosis, calcinosis, acro-osteolysis, RP, and DU (Fig. 1). The occurrence of these various hand manifestations can depend on whether patients have been classified as diffuse cutaneous systemic sclerosis (dcSSc) or limited cutaneous systemic sclerosis (lcSSc) and the disease duration. Although there is lack of robust treatment options for hand impairment, recognizing these various manifestations is essential because they can result in reduced hand mobility, dexterity, and grip strength, which can significantly affect occupational activities and activities of daily living (ADL) [1]. Our objective is to discuss the various manifestations, treatment options currently available, and the areas necessitating further investigation for improved management of hand impairment in SSc.

\section{Arthralgias and inflammatory arthritis}

Forty-six to $97 \%$ of patients with SSc will have joint involvement [2]. Joint symptoms may be present prior to the onset of RP or simultaneously [2]. Joints can be affected by arthralgias, inflammatory arthritis, or both. Patients with SSc can have symmetric, polyarticular synovitis of the metacarpophalangeal (MCP) and proximal interphalangeal (PIP) joints in a rheumatoid arthritis (RA)-like pattern, although distal interphalangeal (DIP) joints can also be affected. In the European League Against Rheumatism (EULAR) Scleroderma Trials and 


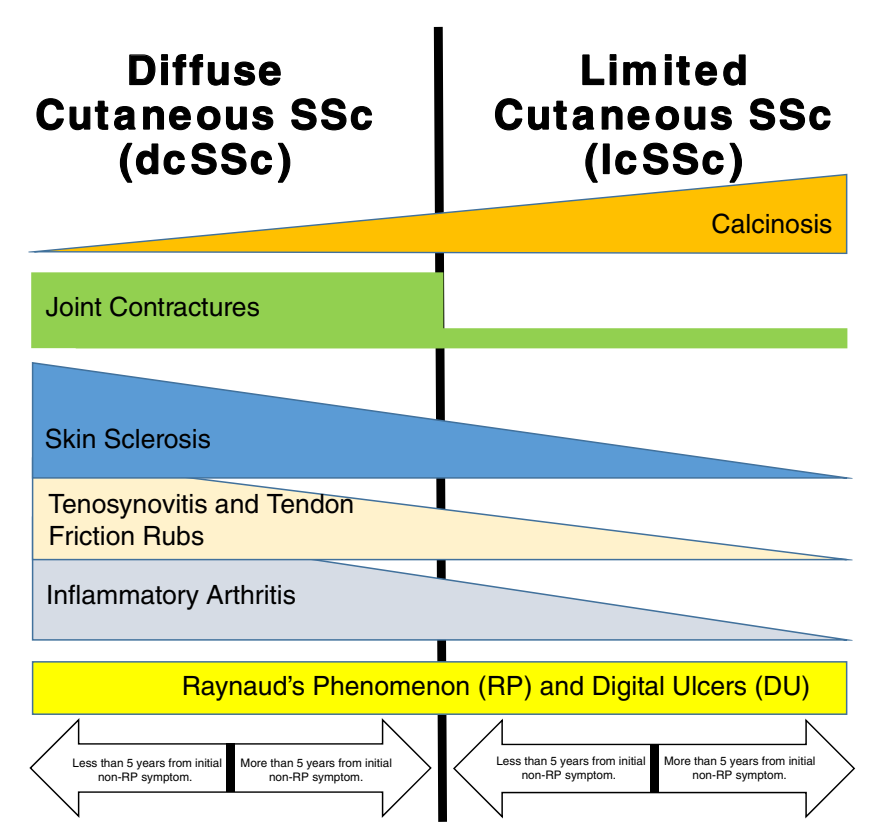

Fig. 1. Comparison of the various manifestations of hand impairment in SSc based on disease subtype and appearance during disease course.

Research (EUSTAR) registry, synovitis had a prevalence of $16 \%$ [3].

Synovitis occurred more often in dcSSc and was a predictive factor for dcSSc, pulmonary hypertension, muscle weakness, new DU, and decreased left heart function $[3,4]$.

Overlap with RA is uncommon in SSc with a prevalence of 1 to $5 \%$. Patients with SSc with overlap with RA will often have erosions and joint deformities [5]. Rheumatoid factor (RF) is present in approximately $30 \%$ of patients with SSc but is not specific and does not predict joint involvement in SSc [2]. The presence of anti-cyclic citrullinated peptide (CCP) in SSc is infrequent ranging from 1.5 to $8 \%[3,5]$.

Histopathological evaluation of SSc-associated arthritis from synovial biopsies has revealed inflammation characterized by lymphocytic and plasma cell infiltration, but in later stages of disease, synovial fibrosis can be present [6]. SSc synovium is more benign than RA synovium as it does not tend to proliferate nor create a pannus [6]. Also, synovial fluid analysis reveals normal to modestly elevated leukocyte count, often with less than 2000 cells $/ \mathrm{mm}^{3}$, comprised of mostly mononuclear cells [6].

To further characterize joint involvement in SSc, radiography is often used. Radiographic changes at MCP, PIP, and DIP joints can include joint space narrowing, erosions, intra-articular calcification, juxta-articular osteoporosis/ osteopenia, and subluxation [2,6]. Erosions can appear similar to erosions in RA with MCP and PIP involvement and juxta-articular osteoporosis/ osteopenia; however, erosions in SSc without overlap with RA are often small and discrete and not as destructive. Additional imaging techniques used to characterize arthritis in SSc are ultrasound (US) and magnetic resonance imaging (MRI). Recent studies have shown US and MRI can detect synovitis in SSc not appreciated on clinical exam [7-9]. 


\section{Outcome measures to assess musculoskeletal (MSK) involvement}

There is lack of consensus on measuring disease activity and outcome measurements for MSK involvement in SSc. The Hand Mobility in Scleroderma (HAMIS) test has shown significant correlations with skin involvement of hands and ADL, and it has been shown to be sensitive to change and useful as an outcome measure [10]. The HAMIS test is a nine-item performance-based measure of impairment using different grips and movements to assess finger and wrist mobility. A modified HAMIS using four items is currently being evaluated as well [10]. Health assessment questionnaire-disability index (HAQDI) and Scleroderma HAQ (SHAQ), which has five additional SSc-specific visual analog scales (VAS) compared to HAQ-DI, are measures of disability that assess difficulties with ADL and have been validated in SSc. Cochin Hand Function Scale (CHFS) assesses hand function and has also been validated in SSc $[5,11 \bullet]$. HAMIS administration requires the use of occupational and/or physical therapists for the assessments whereas SHAQ and CHFS are selfreported measures. Disease Activity Score-28 (DAS-28) use as an assessment of arthritis in SSc has also been suggested [6]. However, recent preliminary data from the Prospective Registry of Early Systemic Sclerosis (PRESS) suggest that it may be difficult to evaluate tender joint count and swollen joint count in early $\mathrm{dcSSc}$ as there was poor association of clinical examination and musculoskeletal US findings likely due to overlying skin fibrosis [12]. Efforts are currently ongoing to develop measures of disease activity and outcome measurements for joint involvement in SSc.

\section{Treatment}

Treatment of inflammatory arthritis in SSc is based on symptoms and typically follows the treatment guidelines for RA. Corticosteroids at doses $\leq 10 \mathrm{mg}$ daily can be used for inflammatory arthritis; however, they must be used cautiously in patients who are more at risk for scleroderma renal crisis (SRC), such as patients with early dcSSc and anti-RNA polymerase III antibody $[2,13]$. Methotrexate is commonly used for treatment of inflammatory arthritis. The subcutaneous route of methotrexate may be considered due to GI dysmotility and poor absorption affecting many patients with SSc [2]. Use of anti-tumor necrosis factor (TNF) alpha agents, such as etanercept and infliximab, in observational studies suggested improvement in inflammatory arthritis according to a systematic review on biologic agents for SSc treatment [14, 15•] Tocilizumab and abatacept use in refractory inflammatory polyarthritis have been reported to be effective in a EUSTAR prospective observational study [16]. Also, a small open-label pilot study involving patients with severe and refractory joint involvement has suggested intravenous immunoglobulin (IVIg) may reduce joint pain and tenderness and may improve hand function and quality of life [17]. Additional off-label inflammatory arthritis treatment includes azathioprine and mycophenolate mofetil [13].

\section{Joint contractures}

Joint contractures are common in SSc with a prevalence of $31 \%$ in the EUSTAR registry and often result in functional disability [3]. They can occur due to skin 
sclerosis, peritendinous sclerosis resulting in tendon shortening, and/or joint destruction leading to ankylosis [18]. Large joint contractures are often of interest due to their presence being a predictive factor for SRC [19]. However, small joint contractures often occur in MCP and interphalangeal (IP) joints of the hands and are important due to known associations with dissatisfaction with appearance, social discomfort, and difficulties performing occupational activities and ADL $[3,5,18,20]$. Fixed flexion contractures of the PIP joints are the most common contractures, which can be associated with fixed extension deformities of MCP joints. DIP joints can be affected by flexion contractures, and thumbs can also be affected by adduction contractures [18, 21] (Fig. 2). Skin ulcerations can even occur over the contractures due to increased pressure on the skin in areas of bony prominences and poor blood flow to the skin from scleroderma vasculopathy [22]. Contractures are associated more often with dcSSc and can be present at any stage of disease; however, evidence has suggested contractures initially present in the early years after disease onset [3, 19, 23]. Anti-topoisomerase I antibody is associated with MCP and PIP joint contractures; this association is thought to occur due to severe skin fibrosis [6, 24]. A study evaluating 350 patients with SSc revealed $47 \%$ of patients had contractures of phalanges; patients with contractures of phalanges often had $\mathrm{dcSSc}$ or positive anti-topoisomerase I antibody; and joint contractures of the hand were often associated with esophageal involvement, pulmonary fibrosis, and cardiac involvement [25]. Also, a study evaluating contractures in dcSSc and lcSSc over 3 years suggested an increased number of contractures for those with more skin thickening and elevated inflammatory markers, and also suggested the dominant hand is more affected by contractures and more than four joint contractures of a single hand may indicate a poor prognosis [23].

\section{Treatment}

In the experience of the authors, large joint contractures can sometimes improve with systemic treatment of severe, progressive skin and/or visceral involvement; however, small joint contractures of the hand often remain unchanged despite

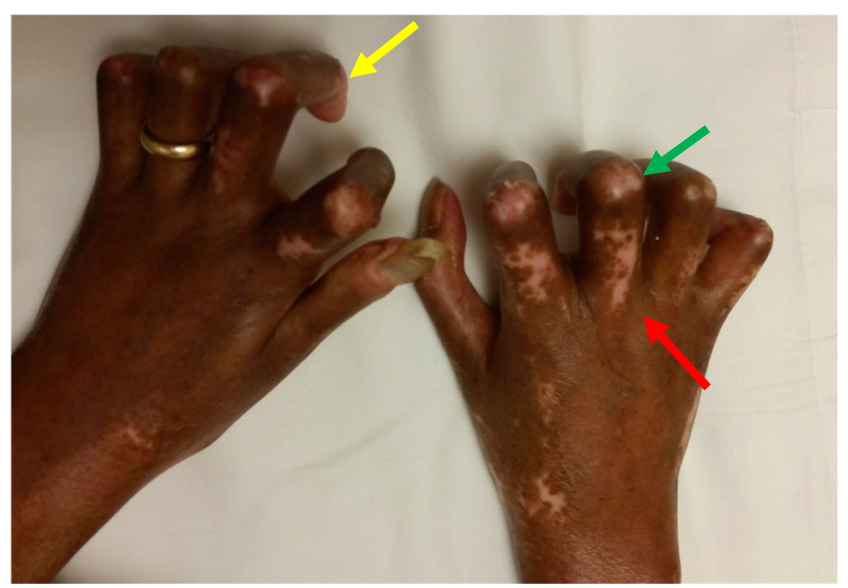

Fig. 2. Hand contractures in SSc (claw hand deformity). Patient with dcSSc with severe contractures including hyperextension of MCP joints (red arrow), flexion contractures of PIP joints (green arrow), and flexion contractures of DIP joints (yellow arrow). 
pharmacologic therapy. Clinical trials for pharmacologic agents are often not evaluating the outcome on contractures of the hand and should be studied in a formal fashion to explore the effects of pharmacologic therapy on small joint contractures of the hand.

Non-pharmacologic therapy can include heat therapy, such as use of paraffin wax, and range of motion (ROM) exercises for the affected joints [26•].

Some patients with severe fixed contractures of the hands can undergo surgery due to pain and/or impaired hand function [18]. Surgical options for patients with contractures may include fusion for DIP and PIP flexion contractures, and capsulotomy or arthroplasty for hyperextension at MCP joints [27]. In a systematic review by Bogoch et al., cross-sectional studies revealed DIP fusions can have uncomplicated wound healing, and patients with moderate to severe hand involvement with PIP contractures and MCP hyperextension can have good outcomes with PIP fusions such as good wound healing, healing of skin ulcerations over PIP joints after surgical correction of joint alone, and radiographic union within 8 weeks post-operatively [18]. However, despite some evidence indicating good outcomes, the benefit of these surgical procedures can be minimal and there can be complications; thus, we do not typically advise surgery for hand contractures, but, if they are performed, they should be performed by an experienced hand surgeon.

\section{Tendon involvement}

\section{Tenosynovitis}

Patients with SSc can be affected by tenosynovitis of the hands. Tenosynovitis has been associated with increased skin score, anti-Scl-70 antibodies, and active and severe disease [7]. Tenosynovitis can be detected by clinical examination, US, and MRI $[7,9]$. In the cross-sectional study on hand US by Elhai et al., tenosynovitis was detected by US in $27 \%$ of patients with SSc compared to detection in only $6 \%$ of patients with SSc based on clinical examination. They also observed two types of tenosynovitis: an inflammatory type associated with power Doppler signal and a sclerosing type characterized by hyperechoic tendon sheath thickening [7]. Sclerosing tenosynovitis appeared to be more specific to SSc than to RA as no RA patients had that type of tenosynovitis [7]. In an observational pilot study of patients with SSc with hand/wrist pain and/or swelling, MRI revealed tenosynovitis in $47 \%$ of patients [9].

Tendon involvement of the hands includes TFRs in extensor and flexor tendons of the digits (Fig. 3). TFRs were initially discovered in a patient with dcSSc in 1876 and recently have been shown to have a prevalence of $11 \%$ according to the EUSTAR registry $[3,4,28]$. They are described as leathery crepitus on palpation of moving joints, which is thought to be due to fibrinous deposits on the surface of tendon sheaths and overlying fascia [2]. TFRs are typically reproducible but have been shown to be intermittent or disappear with repetitive motion [13]. They can also 


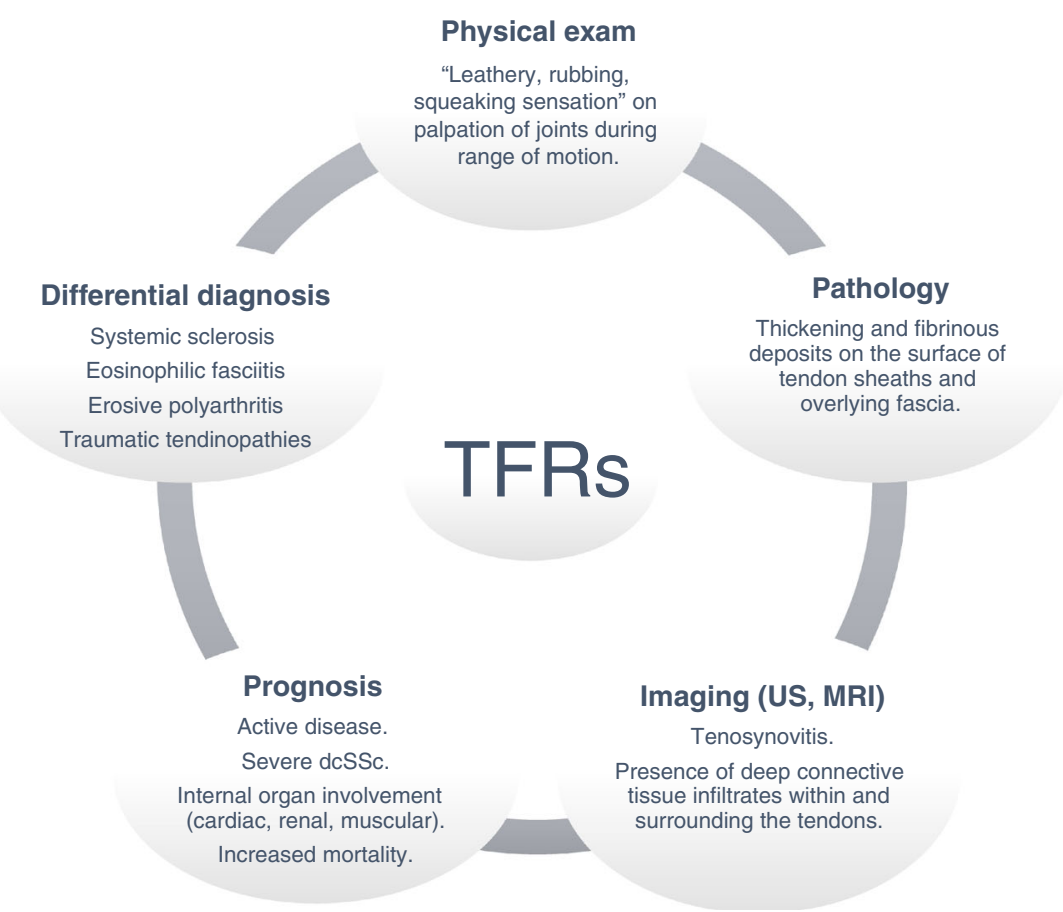

Fig. 3. Importance of TFRs in SSc [2, 28-30, 32].

disappear with immunosuppressive treatment. Tissue pathology has revealed minimal inflammation present in the setting of fibrinous deposits on the surface of tendon sheaths in addition to thickening of tendon sheaths [29]. However, a recent pilot study evaluating involvement of ankles and wrists in dcSSc with MRI suggested TFRs could possibly be due to juxta-tendinous connective tissue infiltrates found in most patients with TFRs, especially since TFRs can be present in tendons without a synovial sheath and over muscles distant from the tendon [30].

Various studies have shown the importance of TFRs as diagnostic and prognostic markers of disease. Medsger and Steen evaluated both dcSSc and lcSSc in a large prevalent observational cohort and found TFRs occurred more often in dcSSc and early disease and were associated with severe skin thickening, heart involvement, renal involvement, joint contractures, and decreased survival [29]. The EUSTAR registry has supported this with significant associations of TFRs with DU, muscle weakness, pulmonary fibrosis, and proteinuria [3]. In a 2-year post-hoc analysis of a randomized controlled trial (RCT) with patients with dcSSc, TFRs were associated with higher HAQ-DI score, and improvement in TFRs was associated with improvement in skin thickness and HAQ-DI [31]. Also, anti-topoisomerase I antibodies are often found in patients with lcSSc and TFRs, which is thought to represent "aborted" dcSSc [3]. A more recent study using TFRs as part of a 5-year mortality tool for risk stratification in patients with early dcSSc revealed that the number of TFRs has a significant weight in risk stratification, which supports the previous literature regarding the importance of TFRs for disease classification, severity, progression, and prognosis [32]. Using advanced radiological 
modalities, Elhai et al. observed that TFRs only occurred in patients with US-proven tenosynovitis, and patients with sclerosing tenosynovitis on US were more likely to have TFRs [7].

\section{Treatment}

Treatment of inflammatory tenosynovitis is similar to treatment of inflammatory arthritis in SSc as mentioned previously with use of agents such as corticosteroid doses $\leq 10 \mathrm{mg}$ daily $[2,33]$. If tendon rupture occurs, surgery may be needed.

\section{Vascular involvement}

\section{Raynaud's phenomenon (RP)}

RP is the most common manifestation of SSc affecting up to $95 \%$ of patients. It is often the initial symptom in patients with SSc. It is characterized by pain, paresthesias, and discoloration of digits due to vasospasm. Classic RP involves three different phases of discoloration: white from ischemia, followed by blue from cyanosis, and then finally red from reperfusion [27].

All patients with RP should avoid vasospastic stimuli such as stress, cold, nicotine, and vasoconstrictive medications. Patients with RP must keep digits covered and warm, particularly in cold environments such as the outdoors and air conditioning. It is also essential for physicians to carefully review medication lists, including over-the-counter agents, to determine if any vasoconstrictive medications are being used. Also, physicians must counsel patients with RP on the importance of smoking cessation and avoidance of second hand smoke [27]. If conservative measures fail to improve RP symptoms alone, pharmacologic therapy may need to be initiated.

There are various pharmacologic agents used for treatment of RP. The initial agent of choice is often calcium channel blockers [34]. According to a systematic review, a meta-analysis of RCTs has supported the use of calcium channel blockers to improve the severity and frequency of RP attacks [35, 36]. Nifedipine has the most evidence supporting its use, but amlodipine is often used [34]. Fluoxetine, a selective-serotonin reuptake inhibitor (SSRI), at a dose of $20 \mathrm{mg}$ daily has been shown to reduce the severity and frequency of RP attacks in a prospective, randomized cross-over pilot study [37]; this agent is often considered for patients with lower blood pressure who become symptomatic with addition of cardiovascular agents for RP treatment. Phosphodiesterase 5 (PDE5) inhibitors have also been beneficial in treatment of SSc-associated RP in various studies. In a meta-analysis of double blind RCTs investigating the treatment of RP with PDE5 inhibitors compared to placebo, a significant benefit with use of PDE5 inhibitors was noted in Raynaud's condition score and the frequency and duration of RP attacks compared to placebo [38]. The following treatments have also been used for RP but there is minimal evidence to support their use: anti-platelet therapy (aspirin or clopidogrel), low molecular weight heparin, topical nitroglycerin, and pentoxifylline [34, 39]. In cases of severe RP, 


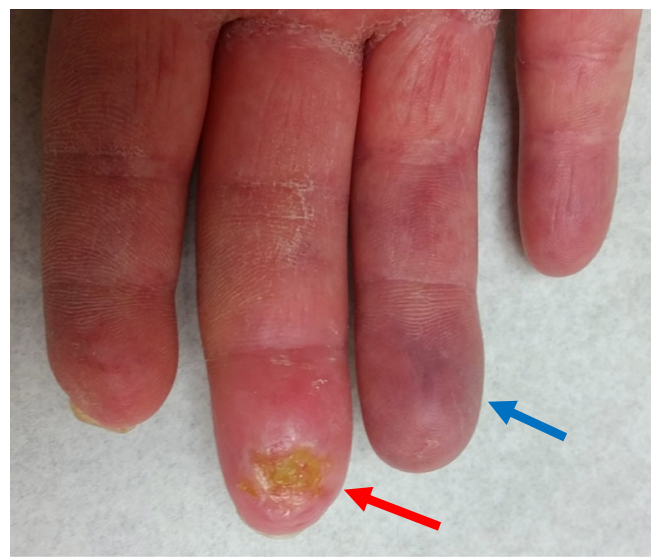

Fig. 4. Vascular complications of SSc. Cyanotic phase of RP (blue arrow). Active digital ulcer (red arrow).

refractory to the previously mentioned agents, intravenous prostacyclins, such as epoprostenol or iloprost, can be used.

RP can be complicated by DU [15•] (Fig. 4). Forty-four to $60 \%$ of patients with SSc have had a DU during their disease course [11 $\bullet$. DU are associated with early onset RP, dcSSc, anti-topoisomerase I antibody, and late and worsening SSc patterns on nailfold videocapillaroscopy [40]. DU can be complicated by infection, gangrene, and amputation. Various studies have shown an association of DU with pain, disability, decreased quality of life, more hospitalizations, progressive cardiovascular disease, and decreased survival $[11 \bullet, 41]$. In a prospective, multicenter, longitudinal study (ECLIPSE), the effect of recurrent ischemic DU on hand disability was assessed in patients who had at least one DU within the previous year and were on treatment with bosentan. The results of this study suggest DU often affect multiple digits on both hands and are significantly associated with pain and hand disability [11 $\bullet$. In a prospective, observational study using the Digital Ulcers Outcome (DUO) registry, four categories of DU were studied over 2 years: no DU, episodic (only one followup visit with DU), recurrent (at least two follow-up visits with DU but one follow-up visit without DU), and chronic (DU present at every follow-up visit) [41]. Results of this study indicate that patients with frequent DU, such as those with recurrent or chronic DU, have a larger disease burden including more complications, need for interventions, and impairment in occupational activities and ADL. Thus, those patients with chronic or recurrent DU have more disease burden and would likely benefit from improved DU management [41].

\section{Treatment}

Non-pharmacologic measures and pharmacologic treatments can prevent and/ or heal DU. To prevent DU, patients should be advised to minimize cold exposure, avoid tobacco use, avoid vasoconstrictive over-the-counter and prescription medication use, and avoid hand injuries, including microtrauma [11•]. Pharmacologic treatment for DU is focused on vasodilatory agents including calcium channel blockers, PDE5 inhibitors, such as sildenafil and 
tadalafil, bosentan, a dual endothelin receptor antagonist, and intravenous prostacyclins, such as epoprostenol or iloprost $[11 \bullet, 42]$. A meta-analysis of 31 RCTs evaluating pharmacologic therapy versus placebo or alternative pharmacologic agents for treatment and/or prevention of DU suggested statistically significant improvement in DU with PDE5 inhibitors, prevention of DU with bosentan, and prevention of digital ulcers with intravenous iloprost [43].

\section{Surgical management of vascular involvement}

For patients with refractory RP with worsening severity and/or frequency of attacks, DU and/or threatened ischemic digits despite use of the previously mentioned pharmacologic and non-pharmacologic treatments, additional procedures may be available for treatment. Botulinum injections have been used in cases of refractory RP [15•, 42]. Also, surgical interventions such as digital sympathectomy or arterial bypass can be considered. A digital sympathectomy consists of adventitial stripping of the common digital arteries, the palmar arch and the ulnar and radial arteries in the wrist. Bypass can involve using grafts to bypass occluded segments of the radial or ulnar arteries [27].

\section{Puffy hands and skin sclerosis of hands}

One of the first non-RP symptoms of SSc is puffy hands due to edema. It is thought that this edematous skin phase is the initial manifestation of SSc prior to skin becoming fibrotic [44]. The appearance of puffy hands is useful in the very early diagnosis of SSc; the presence of RP, puffy hands, and positive antinuclear antibody (ANA) by immunofluorescence should raise suspicion for very early SSc, and diagnosis is confirmed with the presence of SScspecific autoantibodies and/or abnormal nailfold capillaries in a SSc pattern [45].

The skin phase following puffy hands is sclerosis and may last for years. Some patients with lcSSc continue to have puffy hands without progression to sclerodactyly but many do have sclerodactyly present. In later stages of disease, often after 5 years, patients with SSc will start to have thinning of the skin known as the atrophic phase (Fig. 5).

For the early edematous phase, one study has evaluated the use of manual lymph drainage (MLD) for treatment of patients with SSc with edematous hands. It was a randomized controlled study involving 20 patients treated with MLD and 15 patients not treated with MLD for a total of 5 weeks followed by continued observation for 9 weeks with results suggesting improvement in hand edema, hand function, and perceived quality of life [44].

Given the limited skin fibrosis in patients with lcSSc, they usually do not require immunosuppression for treatment of skin sclerosis alone. However, patients with dcSSc, particularly those with early disease, may have progressive, severe skin thickening involving multiple sites requiring treatment with 


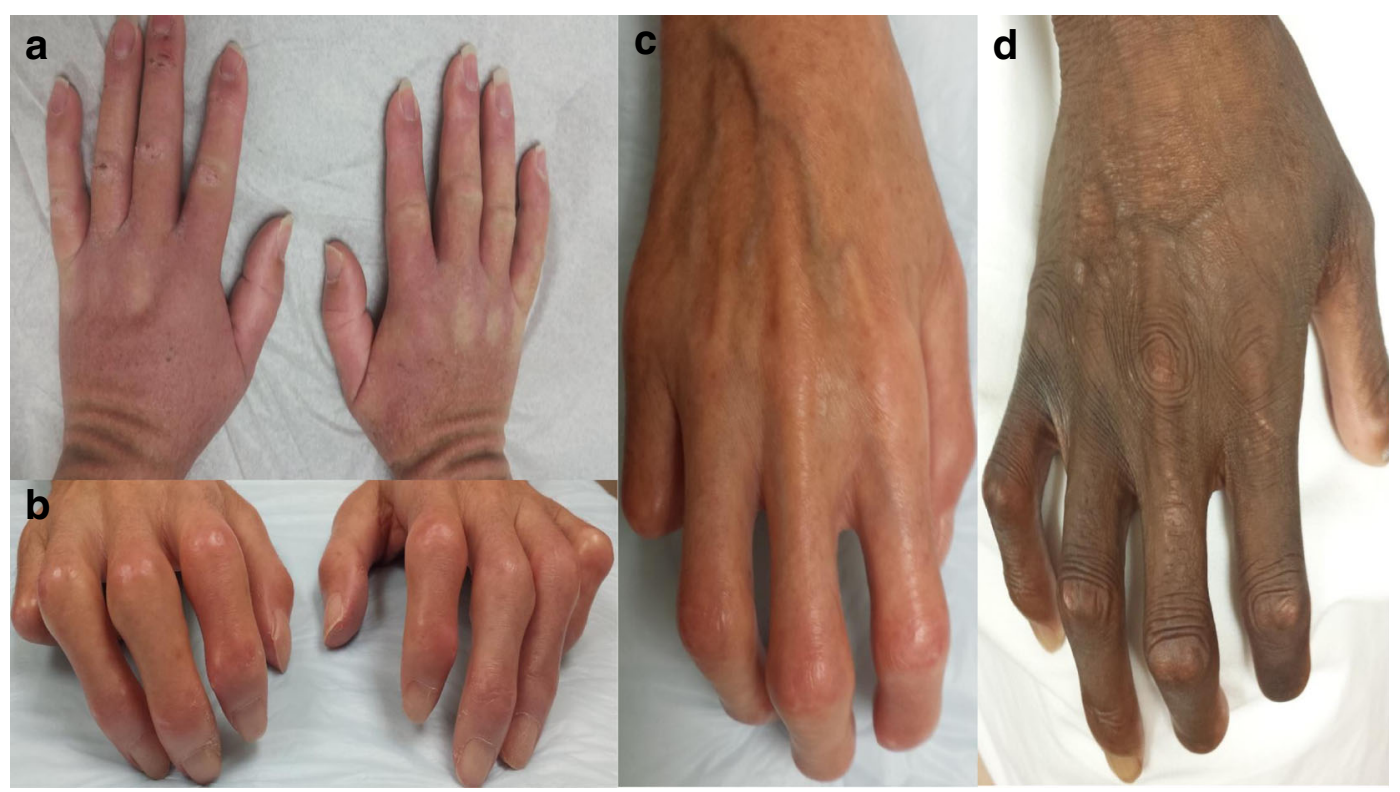

Fig. 5. Phases of skin involvement in SSc. a Initial edematous phase of SSc manifested as puffy hands. $\mathbf{b}$ Sclerodactyly of the digits of the hand due to skin sclerosis resulting in tight skin and loss of skin folds of the fingers. Contractures of PIP and DIP joints are also present in this patient. c A patient with severe dcSSc with areas of atrophy beginning over MCP joints but no appearance of wrinkles, so this is the initial phase of skin atrophy. Contractures of PIP and DIP joints are also present. $\mathbf{d}$ A patient with severe $\mathrm{dcSSc}$ with prior skin thickening but now is in the later stages of atrophy with thin skin and reappearance of wrinkles resembling normal skin. Contractures of PIP and DIP joints are also present.

immunosuppressive agents. The immunosuppressive agent used for skin involvement depends on the presence or absence of inflammatory arthritis and/or visceral organ involvement. In patients with progressive skin thickening, inflammatory arthritis, and no to mild concomitant ILD, we typically use methotrexate, often via subcutaneous route due to GI intolerance, with dosages up to 25 to $30 \mathrm{mg}$ once weekly in our experience. For those patients who did not respond to methotrexate, or they have progressive skin thickening with mild to moderate ILD and no inflammatory polyarthritis, we use mycophenolate mofetil up to $1500 \mathrm{mg}$ twice daily. The duration of immunosuppressive treatment for skin involvement alone is 3 to 5 years, which is based on the natural history of the disease with skin softening often noticeable 5 years after the first non-RP symptom even without treatment.

\section{Acro-osteolysis}

Acro-osteolysis is the resorption of distal phalanges and is associated with acral pulp atrophy (Fig. 6). Resorption of the distal phalanx occurs in about 20 to $25 \%$ of patients with SSc and commonly involves the hands; proximal phalanx bone resorption is rare [5]. When bone resorption of the tuft occurs, it begins on the palmar aspect and leads to a "sharpening" of the phalanx; in some severe cases, all distal digits of the hand can be affected resulting in reduced finger length [5, 19]. The etiology of acro-osteolysis is 

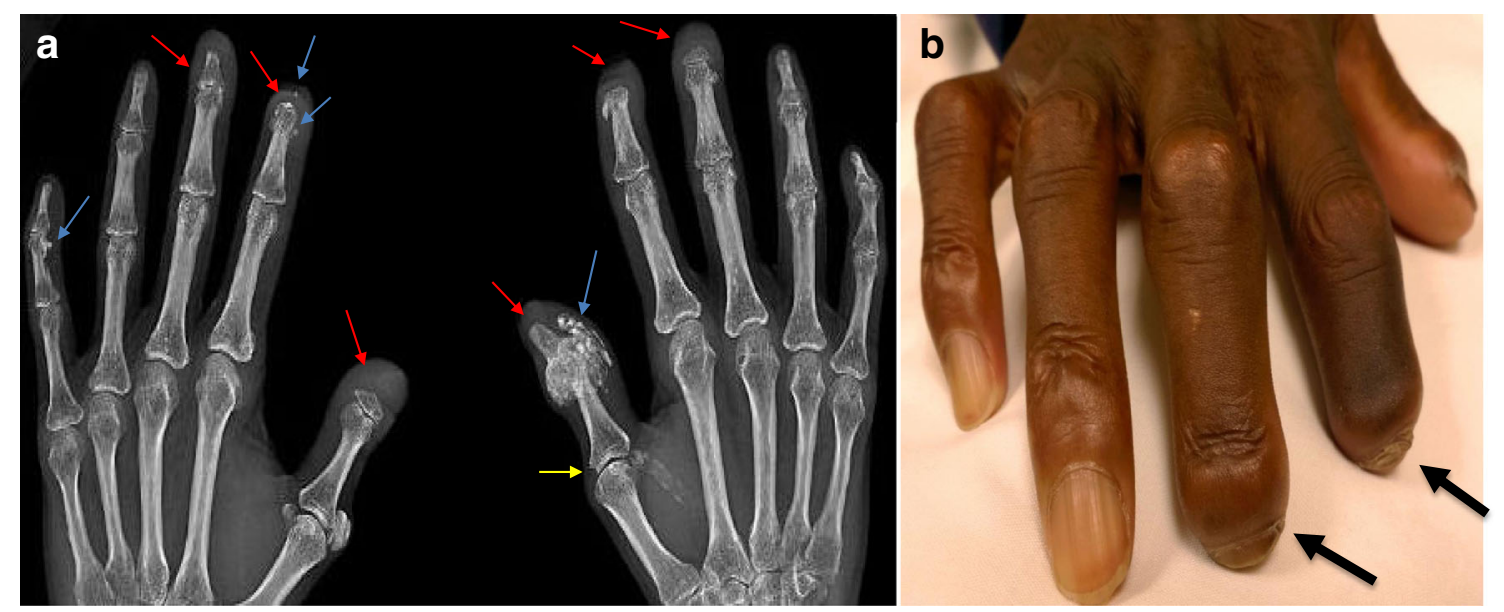

Fig. 6. Acro-osteolysis. a Plain radiographs with resorption of bilateral 1st, 2nd, and 3rd distal phalanxes (red arrows). Also areas of calcinosis (blue arrows) and erosions (yellow arrow) noted. b Acro-osteolysis can be seen on physical examination here with loss of the 2nd and 3rd distal phalanges (arrows).

unclear. It is thought to be due to ischemic atrophy from vasculopathy and retractile pressure from skin thickening [5]. Acro-osteolysis has been associated with severe disease and vascular associations such as calcinosis, DU, and PAH $[13,19,46]$. For example, in a retrospective study, patients with moderate or severe acro-osteolysis were more likely to have severe digital ischemia, and patients with moderate or severe acro-osteolysis were more likely to have severe calcinosis in hand radiographs, but statistical significance for calcinosis was lost after adjusting for potential confounders [47]. Acro-osteolysis can be detected on physical examination and/or with radiological imaging. Studies have shown similar sensitivity of US compared to radiography in detection of acro-osteolysis [13].

\section{Calcinosis}

Calcinosis is caused by the deposition of insoluble calcium salts in skin and subcutaneous tissues, often in areas of frequent microtrauma [5, 48 $]$. It occurs in about $25 \%$ of patients with SSc and can occur in any subset of SSc but is often seen in lcSSc [5, 48 $\bullet$. It commonly involves the hands, often on the palmar aspect of digits in areas of stress. Calcinosis can result in pain, local inflammation, skin ulceration, infection, and impaired hand function, which can ultimately affect quality of life [5, 19, 48•] (Fig. 7). Patients with calcinosis tend to be older with longer disease duration from initial non-RP symptom and often have DU, RP, telangiectasias, acroosteolysis, erosions, arthritis, osteoporosis, cardiac involvement, pulmonary hypertension, and GI involvement [19, 48•, 49]. Some studies have suggested an association with the presence of anti-centromere antibody, antiPM-Scl antibody, and anti-cardiolipin antibodies [48 $\bullet$. Other than detection by palpation on physical exam, calcinosis can be detected by plain radiography, which is very sensitive [5, 48 $]$ (Fig. 7). Additional types of imaging that can evaluate calcinosis include computed tomography (CT) imaging, such as multidetector and dual-energy CT, MRI, and US [48•]. 

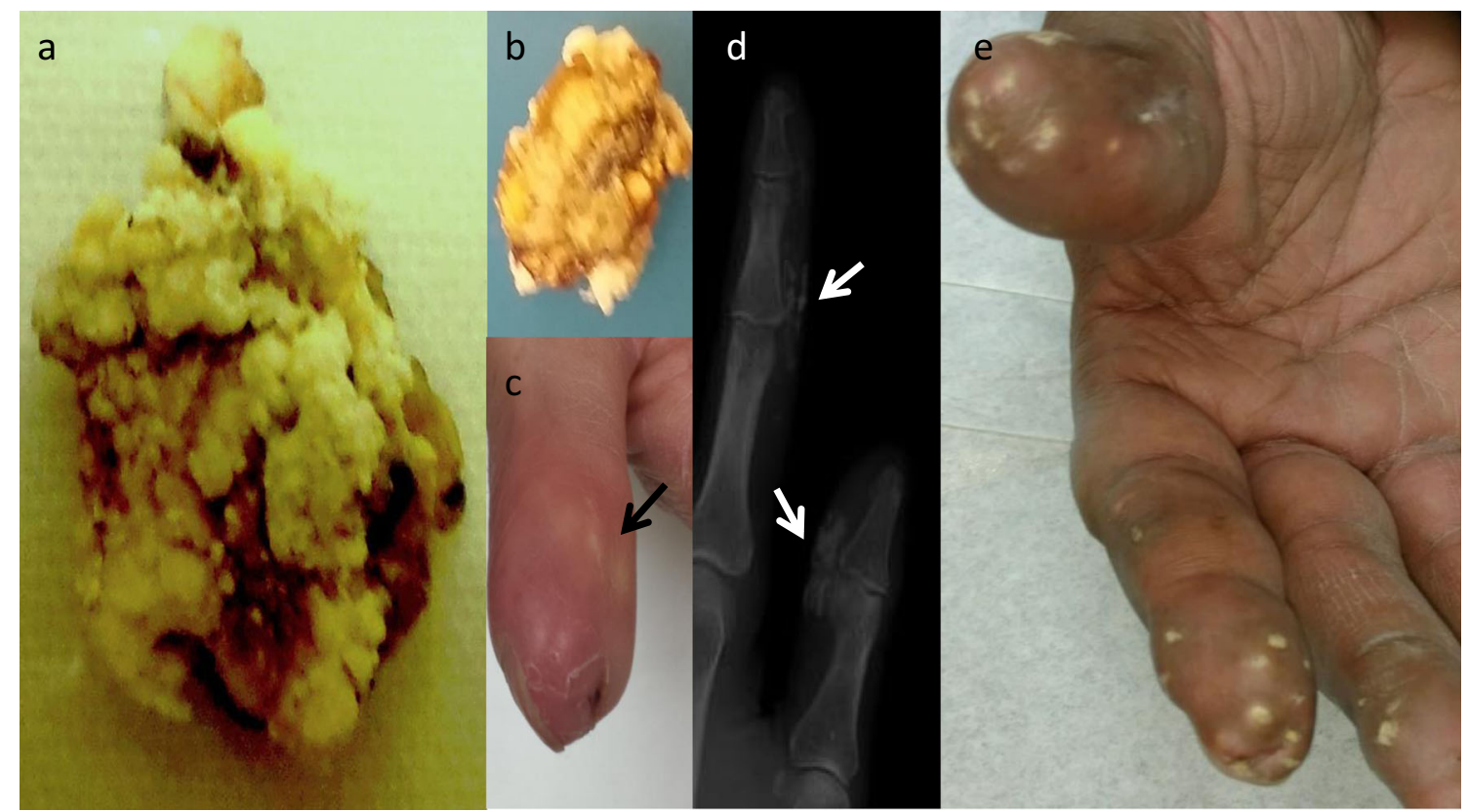

Fig. 7. Calcinosis. a, b Extruded calcium salts from the digits of a patient with calcinosis. c Calcinosis with associated skin breakdown on the 1st digit of the hand (arrow). $\mathbf{d}$ Calcinosis on radiographs near the IP joint and 2nd PIP of the left hand (arrows). $\mathbf{e}$ Calcinosis involving 2 nd digit of the right hand (arrow).

Conservative treatment of calcinosis includes methods to improve blood flow, prevent cold exposure, and avoid trauma, smoking, and stress. Symptomatic treatment of calcinosis includes pain control, antibiotics for infections, and wound care [48•]. According to a review by Valenzuela et al., pharmacologic treatment of calcinosis has been considered and includes agents such as colchicine, minocycline, diltiazem, bisphosphonates, warfarin, probenecid, aluminum hydroxide, IVIg, infliximab, and rituximab [48•]. However, overall, pharmacologic treatment is generally ineffective for calcinosis in SSc. Surgical intervention can be considered for areas of non-inflammatory calcinosis for treatment of pain, functional impairment, and/or skin ulceration due to calcinosis. Various procedures can be performed such as direct excision of focal lesions, fragmentation of lesions subcutaneously followed by flushing out with saline, carbon dioxide laser therapy, or extracorporeal shock wave lithotripsy [18, 49].

\section{Non-pharmacologic management of overall hand impairment in SSc}

Various manifestations can coexist and contribute to the overall hand impairment experienced by patients with SSc; thus, referral to hand 
occupational therapy should be considered. Most therapies focus on the joints of the hand for improved joint mobility and hand function. ROM exercises should be performed in patients with SSc and should include flexion at MCP joints, extension at PIP and DIP joints, opposition of the thumb, and abduction of the digits [26•]. To perform these ROM exercises, the patient applies pressure with one hand to stretch the joints in the opposite hand to the point of skin blanching [26•]. Heat is often used prior to ROM exercises to decrease pain and improve flexibility of collagen [26•]. In a pilot study by Sandqvist et al., use of paraffin wax followed by exercise was compared to exercise alone with results suggesting more improvement in mobility, self-perceived stiffness, and selfperceived skin elasticity in the paraffin wax followed by exercise group compared to the exercise alone group [50]. A case series investigating the use of paraffin wax prior to active exercise has also suggested an improvement in hand function and participation in activities [51].

Splinting has been evaluated, but was not found to be useful [52]. Studies evaluating self-management through home exercises have suggested a benefit in areas of hand pain, hand function, fatigue, and depression [53]. A combination of connective tissue massage, joint manipulation, and home exercise have shown improvement in joint mobility, hand function, and quality of life when compared to daily home exercise alone [26•,54]. However, many of these studies have small sample sizes and short duration of follow-up [26•]. Also, all patients with SSc with impaired hand function should be informed of the availability of various devices to assist with their ADL such as modified handles on kitchen utensils, jar opening devices, button hooks, and modified toothbrushes such as electric toothbrushes [26•].

Novel alternative therapeutic strategies are being investigated as well. In an open-label pilot study of 15 patients with SSc with a non-healing DU despite systemic and local treatment, autologous adipose tissuederived cell fractions were injected into the affected finger; the cardinal DU healed in all patients with a mean healing time of approximately 4 weeks, the DU healing was maintained through the 6-month follow-up, and no new DU developed [55]. A recent open-label, phase I clinical trial called SCLERADEC (NCT01813279) evaluated safety, tolerability, and potential efficacy of the injection of autologous adipose-derived stromal vascular fraction (ADSVF) into the fingers of 12 patients with SSc with hand disability; although this study had a small number of patients and evaluated patients after only 6 months, results do suggest use of ADSVF was safe, well tolerated, and beneficial in terms of hand pain and edema, $\mathrm{RP}$, hand disability, and quality of life [56]. The same cohort of patients was evaluated after 12 months of therapy with ADSVF with results suggesting continued benefits for 1 year [57 $\bullet$. ADSVF is currently undergoing a phase 2, double blind, randomized, placebo-controlled clinical trial called SCLERADEC2 (NCT02558543). The Scleroderma Treatment With Celution Processed Adipose Derived Regenerative Cells (STAR) trial (NCT02396238) is also an ongoing prospective, randomized, multicenter device trial to assess the safety and efficacy of subcutaneous administration of the Celution device processed adipose-derived regenerative cells into the fingers of patients with hand dysfunction due to scleroderma. 


\section{Conclusions}

Hand impairment in SSc is often multifactorial and can be influenced by articular, vascular, skin, and metabolic abnormalities. Most of the published data on hand impairment is focused on DU and the impact on hand disability. Treatment is currently based on individual manifestations of SSc that affect the hand. There is lack of uniform management of the various, often simultaneous, manifestations of hand impairment in SSc. Thus, there is need for improved outcome measures to design future investigations to assess treatment modalities for hand impairment in SSc [5].

\section{Compliance with Ethical Standards}

\section{Conflicts of Interest}

$\mathrm{AY}, \mathrm{RN}$, and CD declare that they have no conflicts of interest. DK reports a consultancy relationship with Bayer, Biogen Idec, Bristol Myers Squibb, Cytori, EMD Serono, Genentech/Roche, Gilead, Glaxo SmithKline, InterMune, Lycera, Medac, Sanofi-Aventis/Genzyme, and Seattle Genetics, and research funding from Scleroderma Foundation and Pulmonary Hypertension Association.

\section{Human and Animal Rights and Informed Consent}

All reported studies/experiments with human or animal subjects performed by the authors have been previously published and complied with all applicable ethical standards (including the Helsinki declaration and its amendments, institutional/national research committee standards, and international/national/institutional guidelines).

\section{Financial Support and Sponsorship}

AY reports salary support from the U.S. Department of Veterans Affairs. RN reports support by NIH/NIAMS grant T32-5T32AR007080-37. DK reports support by NIH/NIAMS grant K24 AR063120.

\section{References and Recommended Reading}

Recently published articles of interest are highlighted as follows:

- Of importance

1. Sandqvist G, Eklund M, Akesson A, Nordenskiold U. Daily activities and hand function in women with scleroderma. Scand J Rheumatol. 2004;33(2):102-7.

2. Avouac J, Clements PJ, Khanna D, Furst DE, Allanore Y. Articular involvement in systemic sclerosis. Rheumatology. 2012;51(8):1347-56. doi:10.1093/ rheumatology/kes041.

3. Avouac J, Walker U, Tyndall A, Kahan A, MatucciCerinic M, Allanore Y, et al. Characteristics of joint involvement and relationships with systemic inflammation in systemic sclerosis: results from the EULAR Scleroderma Trial and Research Group
(EUSTAR) database. J Rheumatol. 2010;37(7):1488-501. doi:10.3899/jrheum. 091165.

4. Avouac J, Walker UA, Hachulla E, Riemekasten G, Cuomo G, Carreira PE, et al. Joint and tendon involvement predict disease progression in systemic sclerosis: a EUSTAR prospective study. Ann Rheum Dis. 2016;75(1):103-9. doi:10.1136/annrheumdis-2014205295.

5. Morrisroe KB, Nikpour M, Proudman SM. Musculoskeletal manifestations of systemic sclerosis. Rheum Dis Clin North Am. 2015;41(3):507-18. doi:10.1016/ j.rdc.2015.04.011. 
6. Randone SB, Guiducci S, Cerinic MM. Musculoskeletal involvement in systemic sclerosis. Best Pract Res Clin Rheumatol. 2008;22(2):339-50. doi:10.1016/j.berh. 2008.01.008.

7. Elhai M, Guerini H, Bazeli R, Avouac J, Freire V, Drape $\mathrm{JL}$, et al. Ultrasonographic hand features in systemic sclerosis and correlates with clinical, biologic, and radiographic findings. Arthritis Care Res. 2012;64(8):1244-9. doi:10.1002/acr.21668.

8. Abdel-Magied RA, Lotfi A, AbdelGawad EA. Magnetic resonance imaging versus musculoskeletal ultrasonography in detecting inflammatory arthropathy in systemic sclerosis patients with hand arthralgia. Rheumatol Int. 2013;33(8):1961-6. doi:10.1007/ s00296-013-2665-8.

9. Low AH, Lax M, Johnson SR, Lee P. Magnetic resonance imaging of the hand in systemic sclerosis. J Rheumatol. 2009;36(5):961-4. doi:10.3899/jrheum.080795.

10. Sandqvist G, Nilsson JA, Wuttge DM, Hesselstrand R. Development of a modified hand mobility in scleroderma (HAMIS) test and its potential as an outcome measure in systemic sclerosis. J Rheumatol. 2014;41(11):2186-92. doi:10.3899/jrheum.140286.

11. Mouthon L, Carpentier PH, Lok C, Clerson P, Gressin $\mathrm{V}$, Hachulla E, et al. Ischemic digital ulcers affect hand disability and pain in systemic sclerosis. J Rheumatol. 2014;41(7):1317-23. doi:10.3899/jrheum.130900.

Prospective, multicenter study evaluating the functional effect of SSc-related DU with results indicating an association of DU with pain and hand disability.

12. Gordon JK, Berrocal VJ, Girish G, Zhang M, Hatzis C, Assassi S, et al. Reliability and validity of the total joint count and swollen joint count in early diffuse systemic sclerosis [abstract]. Arthritis Rheumatol. 2015;67:suppl 10. http:// acrabstracts.org/abstract/reliability-and-validity-ofthe-total-joint-count-and-swollen-joint-count-inearly-diffuse-systemic-sclerosis/. Accessed December 8, 2015.

13. Lorand V, Czirjak L, Minier T. Musculoskeletal involvement in systemic sclerosis. Presse Med. 2014;43(10 Pt 2):e315-28. doi:10.1016/j.lpm.2014. 03.027.

14. Phumethum V, Jamal S, Johnson SR. Biologic therapy for systemic sclerosis: a systematic review. J Rheumatol. 2011;38(2):289-96. doi:10.3899/jrheum.100361.

15. Young A, Khanna D. Systemic sclerosis: a systematic review on therapeutic management from 2011 to 2014. Curr Opin Rheumatol. 2015;27(3):241-8. doi:10.1097/BOR.0000000000000172.

A systematic review discussing the pharmacologic and nonpharmacologic management of systemic sclerosis.

16. Elhai M, Meunier M, Matucci-Cerinic M, Maurer B, Riemekasten G, Leturcq T, et al. Outcomes of patients with systemic sclerosis-associated polyarthritis and myopathy treated with tocilizumab or abatacept: a EUSTAR observational study. Ann Rheum Dis. 2013;72(7):1217-20. doi:10.1136/annrheumdis2012-202657.
17. Nacci F, Righi A, Conforti ML, Miniati I, Fiori G, Martinovic $\mathrm{D}$, et al. Intravenous immunoglobulins improve the function and ameliorate joint involvement in systemic sclerosis: a pilot study. Ann Rheum Dis. 2007;66(7):977-9. doi:10.1136/ard.2006. 060111.

18. Bogoch ER, Gross DK. Surgery of the hand in patients with systemic sclerosis: outcomes and considerations. J Rheumatol. 2005;32(4):642-8.

19. Avouac J, Guerini H, Wipff J, Assous N, Chevrot A, Kahan A, et al. Radiological hand involvement in systemic sclerosis. Ann Rheum Dis. 2006;65(8):1088-92. doi:10.1136/ard.2005.044602.

20. Jewett LR, Hudson M, Malcarne VL, Baron M, Thombs BD, Canadian Scleroderma Research Group. Sociodemographic and disease correlates of body image distress among patients with systemic sclerosis. PLoS One. 2012;7(3):e33281. doi:10.1371/journal. pone.0033281.

21. Anandacoomarasamy A, Englert H, Manolios N, Kirkham S. Reconstructive hand surgery for scleroderma joint contractures. J Hand Surg. 2007;32(7):110712. doi:10.1016/j.jhsa.2007.06.011.

22. Gilbart MK, Jolles BM, Lee P, Bogoch ER. Surgery of the hand in severe systemic sclerosis. J Hand Surg. 2004;29(6):599-603. doi:10.1016/j.jhsb.2004.03. 013.

23. Balint Z, Farkas H, Farkas N, Minier T, Kumanovics G, Horvath $\mathrm{K}$, et al. A three-year follow-up study of the development of joint contractures in 131 patients with systemic sclerosis. Clin Exp Rheumatol. 2014;32(6 Suppl 86):S-68-74.

24. Radic M, Martinovic Kaliterna D, Ljutic D. The level of anti-topoisomerase I antibodies highly correlates with metacarpophalangeal and proximal interphalangeal joints flexion contractures in patients with systemic sclerosis. Clin Exp Rheumatol. 2006;24(4):407-12.

25. Ashida R, Ihn H, Mimura Y, Jinnin M, Asano Y, Kubo $\mathrm{M}$, et al. Clinical features of scleroderma patients with contracture of phalanges. Clin Rheumatol. 2007;26(8):1275-7. doi:10.1007/s10067-006-0490-0.

26. Poole JL, Macintyre NJ, Deboer HN. Evidence-based management of hand and mouth disability in a woman living with diffuse systemic sclerosis (scleroderma). Physiotherapy Canada Physiotherapie Canada. 2013;65(4):317-20. doi:10.3138/ptc.2012-40.

A case study emphasizing various occupational hand therapy techniques used for hand impairment in systemic sclerosis.

27. Fox P, Chung L, Chang J. Management of the hand in systemic sclerosis. J Hand Surg. 2013;38(5):1012-6. doi:10.1016/j.jhsa.2013.02.012. quiz 7.

28. Rose S, Varga J. Diffuse palpable tendon friction rubs in a patient with seronegative erosive polyarthritis. J Clin Rheumatol. 2010;16(6):300-1. doi:10.1097/RHU. ob013e3181ef6ee1.

29. Steen VD, Medsger Jr TA. The palpable tendon friction rub: an important physical examination finding in patients with systemic sclerosis. Arthritis Rheum. 
1997;40(6):1146-51. doi:10.1002/1529-

0131(199706)40:6<1146::AID-ART19>3.0.CO;2-9.

30. Stoenoiu MS, Houssiau FA, Lecouvet FE. Tendon friction rubs in systemic sclerosis: a possible explanation-an ultrasound and magnetic resonance imaging study. Rheumatology. 2013;52(3):529-33. doi:10.1093/rheumatology/ kes307.

31. Khanna PP, Furst DE, Clements PJ, Maranian P, Indulkar L, Khanna D, et al. Tendon friction rubs in early diffuse systemic sclerosis: prevalence, characteristics and longitudinal changes in a randomized controlled trial. Rheumatology. 2010;49(5):955-9.

doi:10.1093/rheumatology/kep464.

32. Domsic RT, Nihtyanova SI, Wisniewski SR, Fine MJ, Lucas M, Kwoh CK et al. Derivation and external validation of a 5-year mortality prediction rule for patients with early diffuse cutaneous systemic sclerosis. Arthritis Rheumatol. 2015. doi:10.1002/art.39490.

33. Dore A, Lucas M, Ivanco D, Medsger Jr TA, Domsic RT. Significance of palpable tendon friction rubs in early diffuse cutaneous systemic sclerosis. Arthritis Care Res. 2013;65(8):1385-9. doi:10.1002/acr.21964.

34. Nagaraja V, Denton CP, Khanna D. Old medications and new targeted therapies in systemic sclerosis. Rheumatology. 2015;54(11):1944-53. doi:10.1093/ rheumatology/keu285.

35. Thompson AE, Shea B, Welch V, Fenlon D, Pope JE. Calcium-channel blockers for Raynaud's phenomenon in systemic sclerosis. Arthritis Rheum.

2001;44(8):1841-7. doi:10.1002/1529-

0131(200108)44:8<1841::AID-ART322>3.0.CO;2-8.

36. Garcia de la Pena Lefebvre P, Nishishinya MB, Pereda CA, Loza E, Sifuentes Giraldo WA, Roman Ivorra JA, et al. Efficacy of Raynaud's phenomenon and digital ulcer pharmacological treatment in systemic sclerosis patients: a systematic literature review. Rheumatol Int. 2015;35(9):1447-59. doi:10.1007/s00296-015-32411.

37. Coleiro B, Marshall SE, Denton CP, Howell K, Blann A, Welsh KI, et al. Treatment of Raynaud's phenomenon with the selective serotonin reuptake inhibitor fluoxetine. Rheumatology. 2001;40(9):1038-43.

38. Roustit M, Blaise S, Allanore Y, Carpentier PH, Caglayan E, Cracowski JL. Phosphodiesterase-5 inhibitors for the treatment of secondary Raynaud's phenomenon: systematic review and meta-analysis of randomised trials. Ann Rheum Dis.

2013;72(10):1696-9. doi:10.1136/annrheumdis2012-202836.

39. Wall LB, Stern PJ. Nonoperative treatment of digital ischemia in systemic sclerosis. J Hand Surg. 2012;37(9):1907-9. doi:10.1016/j.jhsa.2012.02.009.

40. Silva I, Almeida J, Vasconcelos C. A PRISMA-driven systematic review for predictive risk factors of digital ulcers in systemic sclerosis patients. Autoimmun Rev. 2015;14(2):140-52. doi:10.1016/j.autrev.2014.10. 009.
41. Matucci-Cerinic M, Krieg T, Guillevin L, Schwierin B, Rosenberg D, Cornelisse P, et al. Elucidating the burden of recurrent and chronic digital ulcers in systemic sclerosis: long-term results from the DUO Registry. Ann Rheum Dis. 2015. doi:10.1136/annrheumdis-2015208121.

42. Young A, Khanna D. Systemic sclerosis: commonly asked questions by rheumatologists. J Clin Rheumatol. 2015;21(3):149-55. doi:10.1097/RHU. 0000000000000232.

43. Tingey T, Shu J, Smuczek J, Pope J. Meta-analysis of healing and prevention of digital ulcers in systemic sclerosis. Arthritis Care Res. 2013;65(9):1460-71. doi:10.1002/acr.22018.

44. Bongi SM, Del Rosso A, Passalacqua M, Miccio S, Cerinic MM. Manual lymph drainage improving upper extremity edema and hand function in patients with systemic sclerosis in edematous phase. Arthritis Care Res. 2011;63(8):1134-41. doi:10.1002/acr.20487.

45. Avouac J, Fransen J, Walker UA, Riccieri V, Smith $\mathrm{V}$, Muller C, et al. Preliminary criteria for the very early diagnosis of systemic sclerosis: results of a Delphi Consensus Study from EULAR Scleroderma Trials and Research Group. Ann Rheum Dis. 2011;70(3):476-81. doi:10.1136/ard.2010. 136929.

46. Avouac J, Mogavero G, Guerini H, Drape JL, Mathieu A, Kahan A, et al. Predictive factors of hand radiographic lesions in systemic sclerosis: a prospective study. Ann Rheum Dis. 2011;70(4):630-3. doi:10.1136/ard.2010. 134304.

47. Johnstone EM, Hutchinson CE, Vail A, Chevance A, Herrick AL. Acro-osteolysis in systemic sclerosis is associated with digital ischaemia and severe calcinosis. Rheumatology. 2012;51(12):2234-8. doi:10.1093/ rheumatology/kes214.

48. Valenzuela A, Chung L. Calcinosis: pathophysiology and management. Curr Opin Rheumatol. 2015;27(6):542-8. doi:10.1097/BOR. 0000000000000220 .

A recent review on available treatment for calcinosis, which indicates pharmacologic agents have variable outcomes, and surgical excision is a key treatment option for calcinosis.

49. Chander S, Gordon P. Soft tissue and subcutaneous calcification in connective tissue diseases. Curr Opin Rheumatol. 2012;24(2):158-64. doi:10.1097/BOR. ob013e32834ff5cd.

50. Sandqvist G, Akesson A, Eklund M. Evaluation of paraffin bath treatment in patients with systemic sclerosis. Disabil Rehabil. 2004;26(16):981-7. doi:10.1080/ 09638280410001702405.

51. Mancuso T, Poole JL. The effect of paraffin and exercise on hand function in persons with scleroderma: a series of single case studies. J Hand Ther. 2009;22(1):71-7. doi:10.1016/j.jht.2008.06.009. quiz 8.

52. Seeger MW, Furst DE. Effects of splinting in the treatment of hand contractures in progressive systemic sclerosis. Am J Occup Ther. 1987;41(2):118-21. 
53. Poole JL, Skipper B, Mendelson C. Evaluation of a mail-delivered, print-format, self-management program for persons with systemic sclerosis. Clin Rheumatol. 2013;32(9):1393-8. doi:10.1007/ s10067-013-2282-7.

54. Bongi SM, Del Rosso A, Galluccio F, Sigismondi F, Miniati I, Conforti ML, et al. Efficacy of connective tissue massage and Mc Mennell joint manipulation in the rehabilitative treatment of the hands in systemic sclerosis. Clin Rheumatol. 2009;28(10):1167-73. doi:10.1007/s10067-009-1216-x.

55. Del Papa N, Di Luca G, Sambataro D, Zaccara E, Maglione W, Gabrielli A, et al. Regional implantation of autologous adipose tissue-derived cells induces a prompt healing of long-lasting indolent digital ulcers in patients with systemic sclerosis. Cell Transplant. 2015;24(11):2297-305. doi:10.3727/

$096368914 X 685636$.
56. Granel B, Daumas A, Jouve E, Harle JR, Nguyen PS, Chabannon C, et al. Safety, tolerability and potential efficacy of injection of autologous adipose-derived stromal vascular fraction in the fingers of patients with systemic sclerosis: an open-label phase I trial. Ann Rheum Dis. 2015;74(12):2175-82. doi:10.1136/annrheumdis2014-205681.

57. Guillaume-Jugnot P, Daumas A, Magalon J, Jouve E, Nguyen PS, Truillet R, et al. Autologous adipose-derived stromal vascular fraction in patients with systemic sclerosis: 12-month follow-up. Rheumatology. 2015. doi:10.1093/rheumatology/kev323.

An example of an encouraging novel-therapeutic approach for hand impairment in SSc using autologous adipose-derived stromal vascular fraction (ADSVF). 